\title{
Dementia: Towards a Perceptual Control Theory Perspective
}

\section{Authors}

Phil McEvoy, John Eden, Lydia Morris, Warren Mansell

Corresponding author: Phil McEvoy

phil.mcevoy@nhs.net

\section{Purpose}

To explore the psychosocial experiences of people living with dementia using a Perceptual Control Theory (PCT) perspective.

\section{Findings}

The paper suggests that people with dementia control their perceptions by using four modes of control: control, automatic, passive observation and imagination.

\section{Limitations / Implications}

The paper highlights how a perceived sense of 'too little' or 'too much' control can create psychological and emotional distress, as people with dementia seek to respond to the changing contextual circumstances of their lives. However, more work needs to be done to establish empirically-supported principles (ESPs) that can assist the development of strategies and devices that can augment the ability of people living with dementia to exercise control that they are looking for.

\section{Originality}

The potential benefits of adopting a PCT perspective to understand the experiences of people living with dementia have only been explored in a relatively superficial way. This paper is a first attempt to develop a more considered analysis.

Keywords: dementia, Perceptual Control Theory, modes of control, perceptual experience, internal conflict, emotional distress 


\section{Introduction}

Psychosocial approaches bridge the paradigmatic divisions between biomedical approaches that tend to explore mental health problems such as dementia through the lens of psychiatric classificatory systems and phenomenological approaches that focus upon subjective, lived experience (Clare et al., 2011; Carey et al., 2014; Engel, 1980). Perceptual Control Theory (PCT) is a psychosocial theory of behaviour developed by William Powers (Powers et al., 1960; Powers, 1973; 2005). The benefits of using PCT to help us understand the transdiagnostic factors that maintain a range of common mental health problems have been explored in a series of books and review papers over the past decade (Alsawy et al., 2014; Carey, 2006; Carey, 2011; Higginson et al., 2011; Mansell, 2005; Mansell et al., 2012;). However, up until now the potential benefits of adopting a PCT perspective to understand the experiences of people living with dementia have only been explored in a relatively superficial way (McEvoy \& Plant, 2014). This paper is a first attempt to develop a more considered analysis. The paper is divided into four sections: the first section of the paper provides a brief outline of the main principles of PCT; the second section explores how people with dementia monitor and control their perceptual experiences; and the third section examines the dynamics of their social interactions from a PCT perspective. The final discussion and concluding remarks highlight the implications that PCT may have for our understanding of the experiences of people with dementia and makes some suggestions about the need for future research.

\section{Perceptual Control Theory}

Powers developed PCT by using principles from control engineering to analyse human behaviour. After modelling and empirically testing a wide range of behaviours, Powers concluded that all behaviour is purposeful and geared towards the control of our perceptions.(Carey, 2011). He argued that although it may seem to be the case, we do not act instinctively in a stimulus-response manner, 
using our perceptions to guide and control our behaviour. Instead, we consciously or unconsciously compare how things seem to be with how we perceive that they should be and take actions to counteract changes in the perceptual variables that are important to us (Mansell, 2011; Powers, 1998; Yin, 2013).

The fundamental premise on which PCT is based is that people act to control their internal states in order to bring their perceptions in line with their preferred goal states or reference values. PCT proposes that our control systems consist of negative feedback loops, via which we monitor our sensory perceptions, compare our perceptions with our preferred reference points, and act to reduce discrepancies as they arise. Figure 1 illustrates how a simplified negative feedback loop operates. It has five elements: (a) the input - a functional representation that identifies the apparent state of the perceptual variable that is controlled; (b) the reference value - the standard setting or intended state for the perceptual variable; (c) the comparator calculates the difference between the functional representation of the incoming perception with the reference point; (d) the output - the response to deviations from the reference value that are initiated to reduce the error; and (e) feedback - the impact of the output, which interacts with disturbances in the external environment to alter the input. For example, in a noisy café the input is the representation of the level of noise. An individual's reference value could be 'make myself heard'; the comparator would establish the required volume to achieve this reference value (or goal). If a noisy group leaves, this changed input will affect the difference from the individual's reference value, and they will reduce their volume (output). The feedback determines whether the volume of their voice is now enough to 'make themselves heard' and if not they may increase the volume.

PCT suggests that these control systems have many levels. Our higher level control systems tend to relate to abstract things like our personal values and standards for how we want things to be, such 
as doing the right thing by offering to help to wash the dishes. Lower level control systems, control for more concrete things such as the practical steps that are necessary to wash the dishes. These control systems are thought to be arranged in a hierarchical order (Powers, 1973). This enables the higher level control systems to maintain control by setting the reference points for lower level control systems which are able to respond to change more quickly (Yin, 2013).

Rigid or inflexible forms of control that cannot be sustained can produce psychological and emotional distress due to the internal conflict it can generate (Mansell, 2005). The way in which conflicts arise can be explained using a simple example. Imagine yourself sat in a chair reading this paper from a badly positioned desk top computer screen. You might be unaware of the control that you are exerting over your physical posture. However, if you started to experience discomfort in your lower back, it is likely that you would look to alleviate it. This would not be a problematic conflict unless you became so engrossed in the paper that you ignored your rising discomfort. This example illustrates how the ordinary conflicts that we experience in everyday life are maintained. When we are committed to incompatible goals, it is more likely that we will try to control for one of them at the expense of the other in an arbitrary way.

Conflict is an inevitable part of life that we all experience. However, the psychological and emotional distress that we associate with dementia may signal the more serious difficulties that a person with dementia is encountering. This point was illustrated by Robert Davis when he described the impact that his journey into Alzheimer's Disease had upon his ability to maintain the conversational interactions that he had been used to.

"There are times when I feel normal. At other times I cannot follow what is going on around me; as the conversation whips too fast from person to person and before I have processed one comment, the thread has moved to another person or another topic, and I am left isolated from the action- 
alone in a crowd. If I press myself with greatest concentration to try to keep up, I feel as though something short circuits in my brain." (Davis \& Davis, 1989)

Davis was trying to control his perceptual experiences, within the context of the changing social world in which he lived. We do not claim to have any privileged insights into the things that Davis may have been trying to control for. However, he appeared to be trying to reduce the gap between where he felt himself to be and where he instinctively thought that he should be, in relation to the high level goal of trying to maintain his sense of self and social competence. The distress he was experiencing appeared to be related to the difficulty he was experiencing in achieving the goal state that he sought as the extent of his cognitive impairment became increasingly severe. Davis clearly experienced a rising sense of frustration and anxiety about his inability to sustain the level of concentration that was necessary for him to follow and process conversation and express himself in the way that he was used to. This was having a wider impact on his ability to maintain control over the way he presented himself and interact with others on his own terms.

PCT suggests that conflicts such as this are resolved via a trial and error process known as reorganisation. Reorganisation is a process that requires a change in the parameters of a control system. It occurs in an ongoing and continuous way and can restore and enhance control by minimising discrepancies (Mansell et al., 2012; Powers, 1973) However, for the reorganisation to be effective it must take place at the point in the control system that is driving the higher level conflicts. This is because higher level reference values set the parameters for the lower level systems in the control hierarchy (Carey, 2008; Mansell et al., 2012). For people living with dementia this may be especially problematic. This is because the physical deterioration of the neural infrastructure that supports their high level control processes may compromise their ability to monitor and controltheir perceptual reality. 


\section{Monitoring and Controlling Perceptual Reality}

According to PCT, the default way in which we control our experiences and accommodate to the changes that we identify in our internal perceptual environment is through our behavioural actions. However, in order to develop a fuller understanding of the way in which we control our perceptual experiences, it is also necessary to pay attention to the ways in which we assimilate and use the cognitive and affective information that we process through our memories and imagination (Mansell 2005). Powers (1973) suggests that our memories are accessed via a process called 'associative addressing', whereby an element of the stored information itself acts as a key that selectively enables access to other information that is linked to it. This view of memory differs from the traditional view of memory, which conceptualises memory as a factual warehouse. Whereas the traditional view of memory focuses upon the dependability of our memories couched in terms of the amount and factual accuracy of remembered information (Koriat, 2000), PCT encourages us to focus more directly upon the relationship between memory and purposive doing (see also Neisser, 1996).

From the PCT perspective, the relationship between memory and purposeful action is inevitably linked to the processes through which we monitor and control our perceptual reality through our hierarchically organised perceptual systems. Indeed this is the ideal 'mode' of functioning, and it allows an individual to shift their awareness throughout the hierarchy to fulfil a wide range of everyday goals and resolve any conflict at higher levels. Yet, Powers (1973) also highlighted the role of three other perceptual modes: the automatic mode; imaginative mode; and the passive observation mode. These modes deal with the storage and retrieval of perceptual information in different ways depending on the environmental context. In each of the modes the perceptual reference points in the higher and lower levels of our control hierarchies are connected in different ways. The modes enable us to engage in different types of action, which are vital if we are to assimilate new information and adjust to changing situational demands. 


\section{Automatic Mode}

In the automatic mode, the perceptual reference points that we have in the lower order levels of the control hierarchy remain static and no modification by higher order systems occurs. Automatic processes are efficient and they can run in parallel with other control processes (Moors \& de Houwer, 2006) because they do not require our reference points to be adjusted. The efficient use of this mode of control explains why practiced skills that become embedded in our procedural memories, such as making a cup of tea and the building blocks of conversation small talk are maintained in many people living with mild to moderate dementia whose episodic memory is impaired (Bastin et al., 2010). Harnessing these resources can enable individuals with dementia to live well, despite impairments in new learning that are related to episodic memory changes, as they provide the scaffolding that may help them to control their perceptual experiences (Kitwood, 1997).

The automatic mode is likely to be most adaptive when the reference points governing the mode are quite high up in the hierarchy, such as at the program or principle level, and where pursuing that goal is well practised such that the lower levels of that hierarchy are rich in the perceptual goals that are included. The automatic mode of control can be problematic however, when it does not have the level of flexibility that is necessary to adapt to changing circumstances. This explains why people who are able to make a cup of tea in their own home, for example, may find it difficult, if not impossible to do so, in an unfamiliar environment. It may also explain why scaffolding approaches which may be helpful for individuals with episodic memory impairments, may be unnecessary and even inappropriate for individuals who have a relatively well functioning episodic memory and who face challenges due to other cognitive impairments. It may be much more beneficial to provide an alternative type of scaffold, by asking curious questions about the activity they are engaging in, in 
order to help clarify what their goals are and how they would like to achieve them. Clearly, this would have to be done in an empathic and sensitive way.

\section{Passive Observation Mode}

In the passive observation mode, the higher order levels receive perceptual inputs, without changing their behavioural outputs. This allows us to take in information that will potentially help us to acquire new reference values without having to act on them at that moment in time. It is important to be able to take in information relating to one's goals without necessarily acting on the information immediately. We can benefit from this mode in a variety of contexts, like when we absorb experiences and learn vicariously through the observation of others. There may be a number of important reasons why control cannot be exerted within the current situation. For example, a current action may be more appropriate for a different situation (e.g. talking to a different caregiver), a current action may cause more conflict (e.g. showing one's anger may lead other people to be critical), or a current action may be impeded by communication difficulties.

The capacity that individuals with dementia have to absorb their experiences is frequently underestimated. If we do not appreciate the experiences that someone with dementia is absorbing or fail to read their communications because we perceive them to be meaningless, they may react by acting out their frustrations or withdrawing into silence (Acton et al, 2007). Ward and his colleagues (2008) gave a moving illustration of this important point in a qualitative study that explored the patterns of communication in residential dementia care. Michael, a man with severe dementia, had lost his ability to communicate through speech and his facial expression rarely altered. His ability to communicate the experiences he was absorbing was frequently overlooked by the care staff who looked after him. Despite this, the researchers who conducted the study identified from observations of his non-verbal behaviour that there was a distinct change in his 
demeanour when his wife visited. His movements quickened, as he expressed his excitement, and when she left his movements slowed and sometimes completely ceased, signalling his sadness.

\section{Imagination Mode}

In the imagination mode, not only are the means of implementing goals shortcircuited, but the perceptual input from the environment is bypassed too. This means that the perceptual reference points in the higher levels of the control hierarchy operate completely independently of the lower order levels. Our imagination fills the gaps between what we actually perceive and what we want to perceive and short-circuits the control hierarchy so that the memory of what we want or expect to perceive is transmitted upwards, in the absence of signals from the environment. This can be considered as a form of covert control that operates off-line, using the same sort of mechanisms as overt control. Within this mode, 'as if' perceptions are substituted for sensory perceptions, which are not actually present. The breadth and duration of these 'as if' perceptions can vary. They can be as simple as the insertion of non-existent words into a written text. This effect may approximate to what has been termed 'implicit' memory in cognitive psychology. The imagination mode may also extend to fantasies about the future and the mental simulations that are involved in thinking ahead and predicting the anticipated course of events. Other theoreticians have pointed out that our imagination is not constrained by the realities of spatial location, temporal coherence or material causality and that we can use our imaginations to develop theories of mind about our own mental states and the mental states of others (Fonagy et al., 2011; Sandler et al., 1997). Linguistic studies have shown that people living with dementia may be able use the imagination mode in adaptive ways. For example, some individuals may fill in their speech with formulaic language to maintain the thread of a conversation when they have word finding difficulties (Wray, 2008). 
The imagination mode may be disruptive for some individuals if it is a substitute for perception of the environment, rather than an 'as if' simulation. The problem here is not that they are actively trying to control their perceptual experiences using their imagination. It is that they may be locked into their imagination mode in an inflexible way without being able to easily switch back into the control mode, in order to adjust their responses and deal with their conflicts in more adaptive ways. The imagination mode may then become the fuel for more extreme thinking that goes unchecked within the environment, building up worry and fears. Capgras Syndrome, the belief that a person who is well known to someone has been replaced by an identical imposter, is relatively common in people who have dementia with Lewy Bodies and advanced Parkinson's Disease (Edelstyn et al., 2014; Thaipisutikul et al, 2002). The syndrome is thought to be related to impaired facial recognition and paranoia, and/or anxiety related perceptions that are associated with the misidentified person (Cipriani et al., 2013). Relatives and care workers can find the behaviour of people with Capgras Syndrome difficult to understand. As a consequence, they may look to protect themselves and deal with their own need to maintain a semblance of control by suggesting that an individual is 'living in their own world' and 'cut off' from their environment (Balfour, 2006). Yet, from a PCT perspective, the way in which a person with Capgras Syndrome is using their imagination mode to make sense of their perceptual experiences can be understood as something that could have much more significance than a case of simple mis-recognition or a recall problem. A benefit of this vantage point, it that it may help caregivers to engage with the persons psychic reality more empathically, as they reflect on the person's experiences and how they may be trying to control them.

\section{Dynamics of Interpersonal Interaction}

PCT has not only been applied to the individual, but it has also informed research on interpersonal and social processes. The sociologist Kent McClelland (2006) whose work is guided by the application of PCT suggests that the dynamics of social relationships can be understood through the analysis of control processes. He argues that interpersonal conflict is inevitable, as we do not always succeed in 
controlling our perceptions and because our individual vantage points and perceptions will diverge. Nevertheless McClelland has proposed that the maintenance of our cultural world - language, religion, cities, organisations - depends upon the management of the dynamic tension that arises between competing differences and the need to find forms of collective control. Collective control relies on individuals establishing collaborative goals and overlapping reference values for what they want to perceive. Such an understanding may help to enhance rapport, engagement and collaboration with a person who is living with dementia.

For people living with dementia it can become increasingly difficult to pick up on social cues and make themselves understood (Bougeoius, 2002; Ryan et al., 2005). These challenges can be compounded, if individuals are asked to identify factual references that they cannot recall. For example, the question "Do you remember when we had dinner before?" This may risk creating conflict and an experience of frustration as the person may not be able to access the references due to the difficulties they encounter in recalling information. It is likely to be much more helpful to give the individual an invitation to respond that allows them to work with the reference values they have. This kind of approach can help to avoid triggering the unnecessary embarrassment and threat to their identity which they may experience, when they are aware of their failure to meet social expectations and feel publicly exposed (Ho, 1976). Asking short non-intrusive questions that open up space for people to talk about their present experience or using simple conversational prompts about their experience may also promote collaborative engagement (McEvoy \& Plant, 2014).

Collaboration may be asymmetric in the sense that one person or group may be in a position in which they seem to contribute more than the other, but at a fundamental level collaboration between two or more people always requires two preconditions. Firstly, the recognition and acceptance that the other person or people have an independent perspective and take on reality. Secondly, the creation of a space in which this reality can be explored in a way that allows them to 
exercise the control that they seek (Benjamin, 1988). The aim of a PCT informed approach would be to facilitate engagement in the controlled mode, by proving an environmental context in which people with dementia feel safe and secure and gives them opportunities to share and enact their goals. This may require that carers offer the support that people living with dementia need in order to stay as actively engaged in communal activities as they are able and want to be. The level of this support would also need to be adapted to deal with the constraints that may arise from neurologically driven changes that affect their ability to process information, and the shifting dynamic of their relationships with their carers on whom they may rely (Loboprabhu et al., 2007).

\section{Discussion}

PCT is based upon the position that all behaviour is purposeful and geared towards the control of our perceptual experiences and it starts from an assets based position (Morgan \& Ziglio, 2007), in the way that it approaches the resources that people with dementia have. This is because PCT maintains that all living people are active and intentional agents and it is possible for them to maintain positive wellbeing, as long as they are able to adapt and respond to their contextual circumstances by maintaining the perceptual control that they are looking for. This paper has highlighted how the ability of a person living with dementia to respond and adapt to changing circumstances may be influenced by the dynamic interplay between four different modes of control. These modes of control may play an important role in helping us to understand the ways in which people with dementia monitor and take steps to control their perceptual reality via various modes. A perceived sense of 'too little' or 'too much' control can lead to psychological and emotional distress due to internal conflict it may generate. 
- In the control mode people with dementia accommodate to the changes that occur in their internal perceptions through their behavioural actions. This mode of control is very adaptable but it can also produce internal conflict.

- In the automatic mode people with dementia act without adjusting their perceptual reference values. The automatic mode is efficient but inflexible.

- In the passive observation mode, the perceptual reference values change but there is no change in the behavioural output. The capacity that people with dementia have to passively absorb their experiences may be underestimated.

- In the imagination mode, 'as if' perceptions are substituted for sensory perceptions, which are not actually present. The imagination mode is vital for problem solving and making sense of our experience, but when people with dementia are 'stuck' within their imagination mode their carers may find this difficult to understand.

PCT provides a set of conceptual resources that may assist family and professional carers by pointing towards key questions that they may need to address if they are to empathise and connect with someone with dementia.

- What is the individual communicating to me and what does this tell me about the way that she is trying to control her perceptual experiences?

- Which mode of control may she be engaged in and how might this be influencing the way that they are trying to manage her experiences through her behaviour, communicative demands, thinking, memory or imagination?

- Are there conflicts that may be arising from the competing goals that the individual may have?

- How does my own experience right now as I am interacting with the individual with dementia compare to my preferred goal state? 
- How can I support the person I am caring for by giving her as much control as is feasible without taking unjustifiable risks?

These reflective questions may help carers to develop a more intuitive understanding of the preferred goal states of the person with dementia and how they are employing the different modes of control in order to try and achieve them. If carers are able to tune into the perspective of people with dementia they may be able to able to support their efforts to maintain the control they are looking for. In some cases, this may be scaffolded by relatively simple communication techniques that give the person with dementia an invitation to respond. Family and professional carers could be provided with specific training in such reflective questions; for example, descriptions of when such questions might be particularly pertinent, and the opportunity to share their reflections. These concepts could also provide tools for carers to understand some of the complex perceptual processes that can underlie everyday interactions with individuals with dementia. For example, recognising that even when behavioural output is limited (individuals are in the passive observation mode) internal reference values are likely to be changing, and therefore continuing to maintain communication with the individual and encourage expression where possible.

Yet, we must also be careful not to underestimate these challenges (Balfour, 2006). For people with dementia and their carers, a reason for blocked action may also be the resonance that they have with previously unresolved conflicts. This may subconsciously generate uncomfortable feelings that are very difficult to tolerate or put into words and lead to automatic reactions that are outside of their awareness. A paradox that may also arise is that, in order to facilitate greater communicative engagement and the empowerment of the person with dementia, the carer may have to relinquish the sense of control that they value. This is likely to generate internal conflict and ambivalence. It may only be resolved in a supportive environment that provides them with the back-up they need to realise their goals and aspirations. 


\section{Concluding Remarks}

Given that this paper is a first attempt to develop a considered analysis of the potential benefits of using a PCT perspective to understand the experiences of people living with dementia, it is clearly limited in scope. Much more work more work needs to be done to establish empirically-supported principles (ESPs) that can assist the development of strategies and devices that can augment the ability of people living with dementia to exercise control that they are looking for as the neural infrastructure that supports their high level control systems deteriorates (Kasl-Godley \& Gatz, 2000). It will be necessary to test these principles and trial strategies that are developed in order to assess their effectiveness. This may require a combination of quantitative, qualitative and modelling methodologies in order to explore the complex processes and causal mechanisms that are active in individual cases (Marken \& Carey, 2014; McEvoy \& Richards, 2006). Despite these limitations, the paper has shown how people living with dementia may use their resources and capabilities to control their perceptual experiences via different modes of control. Adopting a PCT perspective has also helped to clarify some of the challenges that people with dementia face as they wrestle with their declining cognitive abilities and changing contextual circumstances of their lives. 


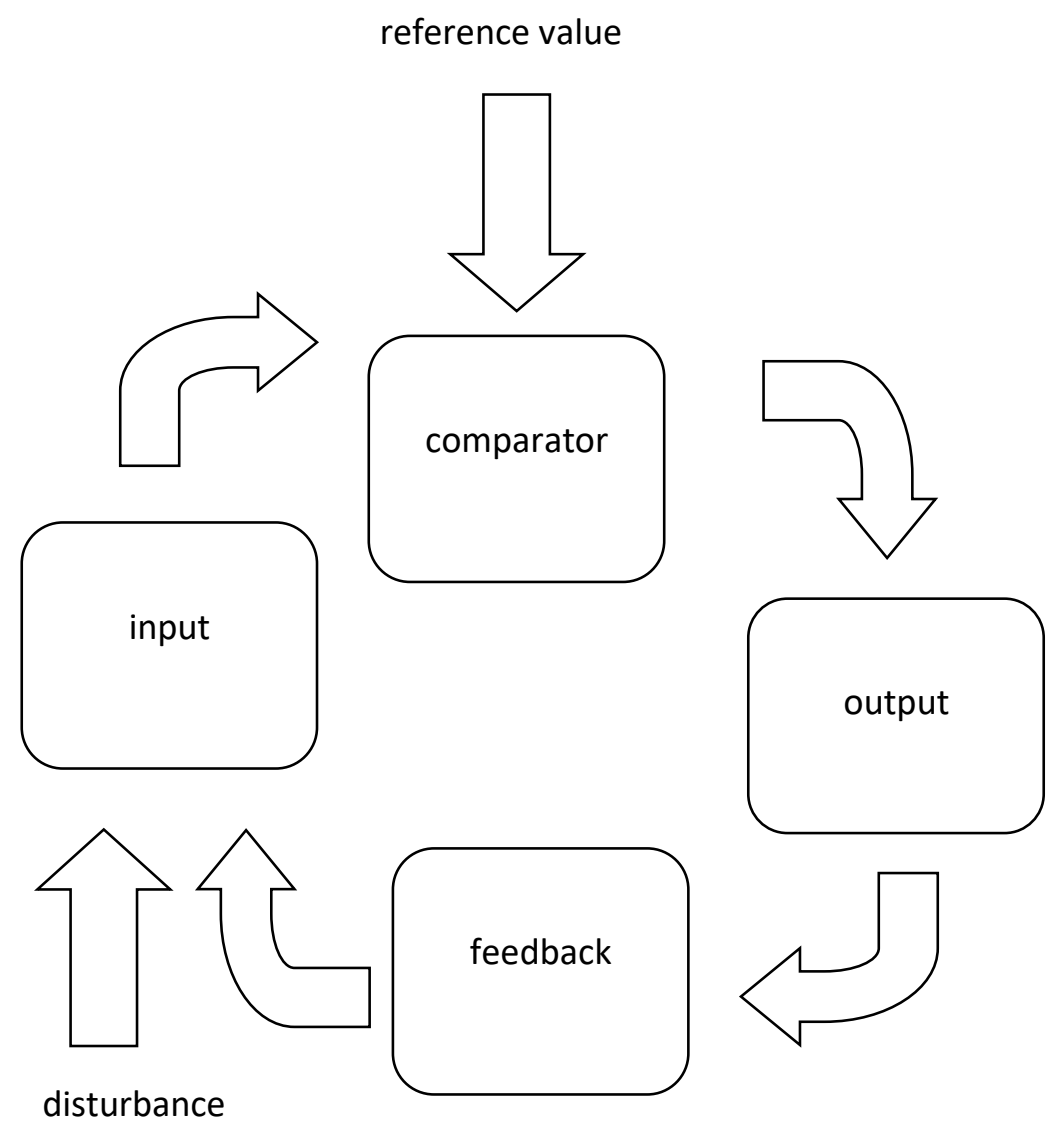

Figure1. Simplified Schema for Negative Feedback Loop (modified from Powers, 1973) 


\section{References}

Acton GJ, Yauk S, Hopkins BA \& Mayhew PA (2007) Increasing social communication in persons with dementia. Research and Theory for Nursing Practice 21 (1) 32-44.

Alsawy S, Mansell W, Carey TA, McEvoy P \& Tai SJ (2014) Science and Practice of Transdiagnostic CBT: A Perceptual Control Theory (PCT) Approach. International Journal of Cognitive Therapy 7 (4) 334-359.

Balfour A (2006) Thinking about the experience of dementia: The importance of the unconscious. Journal of Social Work Practice 20 (3) 329-346.

Bastin C, Kerrouche N, Lekeu F, Adam S, Guillaume B, Lemaire C...Salmon E (2010)Controlled memory processes in questionable Alzheimer's disease: A view from neuroimaging research. Journal of Alzheimer's Disease 20 547-560.

Benjamin J (1988) The Bonds of Love. New York: Pantheon.

Bourgeois MS (2002) "Where Is My Wife and When Am I Going Home?" The Challenge of Communicating with Persons with Dementia. Alzheimer's Care Today 3 (2) 132-144.

Carey T (2006). The method of levels: How to do psychotherapy without getting in the way. Hayward, CA: Living Control Systems Publishing.

Carey TA (2011) Exposure and reorganization: The what and how of effective psychotherapy. Clinical Psychology Review 31 236-248. 
Carey TA, Mansell W, \& Tai SJ (2014) A biopsychosocial model based on negative feedback and control. Frontiers in Human Neuroscience 8 (94) 1-10.

Cipriani G, Vedovello M, Ulivi M, Lucetti C, Di Fiorino A \& Nuti A (2013) Delusional Misidentification Syndromes and Dementia A Border Zone Between Neurology and Psychiatry. American journal of Alzheimer's disease and other dementias 28(7) 671-678.

Clare L, Marková IS, Roth I \& Morris RG (2011) Awareness in Alzheimer's disease and associated dementias: Theoretical framework and clinical implications. Aging \& Mental Health 15 (8) 936-944.

Davis R \& Davis B (1989) My journey into Alzheimer's disease. Wheaton, IL: Tyndale House Publishers.

Edelstyn NMJ, Drakeford J L \& Ellis S J (2014) Visual Hallucinations in Parkinson's Disease: a Hierarchy of Impairments Involving Perception, Source Monitoring and Reasoning. Austin Journal of Psychiatry and Behavioral Science 1 (7) 8.

Engel GL (1980) The clinical application of the biopsychosocial model. American Journal of Psychiatry $137535-544$.

Fonagy P, Bateman A \& Bateman A(2011) The widening scope of mentalizing: A discussion. Psychology and Psychotherapy: Theory, Research and Practice 84 (1) 98-110. 
Higginson S, Mansell W, \& Wood, AM (2011) An integrative mechanistic account of psychological distress, therapeutic change and recovery: The Perceptual Control Theory approach. Clinical Psychology Review 31 (2) 249-259.

Ho DYF (1976) On the Concept of Face. American Journal of Sociology 81 (4) 867-884.

Hydén LC (2014) Cutting Brussels sprouts: Collaboration involving persons with dementia. Journal of Aging Studies 29 115-123.

Kasl-Godley J \& Gatz M (2000) Psychosocial interventions for individuals with dementia: An integration of theory, therapy, and a clinical understanding of dementia. Clinical Psychology Review 20 (6) 755-782.

Kitwood T (1997) Dementia Reconsidered: The Person Comes First. Buckingham: Open University Press.

Koriat A (2000) The feeling of knowing: Some metatheoretical implications for consciousness and control. Consciousness and cognition 9 (2) 149-171.

Loboprabhu S, Molinari V \& Lomax J (2007) The transitional object in dementia: clinical implications. International Journal of Applied Psychoanalytic Studies 4 (2) 144-169.

Mansell W (2005) Control Theory and Psychopathology. An Integrative Approach. Psychology and Psychotherapy: Theory Research and Practice 78 141-178. 
Mansell W (2011) Control of Perception Should be Operationalised as a Fundamental Property of the Nervous System. Topics in Cognitive Science 3 (2) 257-261.

Mansell W, Carey TA, \& Tai SJ (2012) A Transdiagnostic CBT Using Method of Levels Therapy. London: Routledge.

Marken RS \& Carey TA (2014) Understanding the Change Process Involved in Solving Psychological Problems: A Model-based Approach to Understanding How Psychotherapy Works. Clinical Psychology \& Psychotherapy (in press).

McClelland KA (2006) Understanding Collective Control Processes. In KA McClelland \& TJ Fararo (Eds.) Purpose, Meaning, and Action: Control Systems Theories in Sociology (pp 31-56). New York: Palgrave Macmillan.

McEvoy P \& Plant R (2014) Dementia care: using empathic curiosity to establish the common ground that is necessary for meaningful communication. Journal of Psychiatric and Mental Health Nursing 21 (6) 477-482.

McEvoy P \& Richards D (2006) A critical realist rationale for using a combination of quantitative and qualitative methods. Journal of Research in Nursing 11 (1) 66-78.

Moors A \& De Houwer J (2006) Automaticity: A theoretical and conceptual analysis. Psychological Bulleti, 132 297-326.

Morgan A \& Ziglio E (2007) Revitalising the evidence base for public health: an assets model. Promotion and Education S2, 7-22. 
Neisser U (1986) Nested structure in autobiographical memory. In DC Rubin (Ed) Autobiographical Memory (pp71-81). Cambridge: Cambridge University Press.

Powers WT, Clark RK \& McFarland R L (1960) A general feedback theory of human behavior. Part II. Perceptual and Motor Skills 11 309-323.

Powers WT (1973, 2005) Behaviour: The Control of Perception. New Canann, Connecticut : Benchmark Publications Inc.

Powers W T (1989) Living Control Systems. Montclair, NJ: Benchmark Publications.

Ryan EB, Byrne K, Spykerman H \& Orange JB (2005) Evidencing Kitwood's Personhood Strategies: Conversation as Care in Dementia. In BH Davis (Ed), Alzheimer talk, text, and context: Enhancing communication (pp 18 - 36). Basingstoke: Palgrave Macmillan.

SandlerJ, Dare C, Holder A \& Dreher U (1997) Freud's models of the mind: an introduction. London: Karnac.

Thaipisutikul P, Lobach I, Zweig Y, Gurnani A, \& Galvin J E (2002) Capgras Syndrome in Dementia with Lewy Bodies. Journal of Geriatric Psychiatry and Neurology 15 (4) 239.

Ward R, Vass AA, Aggarwal N, Garfield C \& Cybyk B (2008) A different story: exploring patterns of communication in residential dementia care. Ageing and Societ, 28 (5) 629-651.

Wray A (2008) Formulaic language: Pushing the boundaries. Oxford: Oxford University Press. 
Yin H H (2013) Restoring purpose in behavior. In G. Baldassarre \& M. Mirolli (Eds), Computational and robotic models of the hierarchical organization of behavior (pp 319-347). Berlin: Springer-Verlag. 